\title{
Gastroprotective Effect of Myricetin on Ethanol-Induced Acute Gastric Injury in Rats
}

\author{
Hee-seon Park, ${ }^{1}$ Chang-Seob Seo, ${ }^{2}$ Eun Bok Baek, ${ }^{1}$ Jin-hyung Rho, ${ }^{1}$ Young-Suk Won, ${ }^{3}$ \\ and Hyo-jung Kwun $\mathbb{D}^{1}$ \\ ${ }^{1}$ Department of Veterinary Pathology, College of Veterinary Medicine, Chungnam National University, \\ Daejeon 34134, Republic of Korea \\ ${ }^{2}$ Herbal Medicine Research Division, Korea Institute of Oriental Medicine, Daejeon 34054, Republic of Korea \\ ${ }^{3}$ Laboratory Animal Resource Center, Korea Research Institute of Bioscience and Biotechnology, Cheongju, Republic of Korea
}

Correspondence should be addressed to Hyo-jung Kwun; hyojung@cnu.ac.kr

Received 12 March 2021; Revised 23 August 2021; Accepted 25 August 2021; Published 30 September 2021

Academic Editor: Man Hee Rhee

Copyright (C) 2021 Hee-seon Park et al. This is an open access article distributed under the Creative Commons Attribution License, which permits unrestricted use, distribution, and reproduction in any medium, provided the original work is properly cited.

\begin{abstract}
The flavonoid myricetin is abundant in vegetables and has various bioactive properties, including anti-inflammatory and antioxidative activities. In the present study, we explored the effects of myricetin on alcohol-induced gastric ulcer in a rat model. To induce gastric ulcer, absolute ethanol $(5 \mathrm{~mL} / \mathrm{kg}$ body weight) was orally administrated to each rat. The positive control and myricetin-treated groups were given oral doses of omeprazole $(20 \mathrm{mg} / \mathrm{kg})$ or myricetin $(12 \mathrm{mg} / \mathrm{kg})$, respectively, 1 hour prior to the administration of absolute alcohol. We found that pretreatment with myricetin significantly decreased alcohol-induced gastric ulcer, hemorrhage, hyperemia, and epithelial cell loss in the gastric mucosa. Myricetin pretreatment reduced the level of malondialdehyde (MDA) and increased that of total glutathione (GSSG/GSH) and superoxide dismutase (SOD) in gastric tissues. In addition, it elevated the expression levels of cyclooxygenase-1 (COX-1) and prostaglandin $\mathrm{E}_{2}\left(\mathrm{PGE}_{2}\right)$ and decreased the phosphorylation of nuclear factor kappa B (NF- $\kappa \mathrm{B})$. Together, these results indicate that myricetin effectively inhibits ethanolinduced acute gastric injury by preventing oxidative damage, stimulating $\mathrm{PGE}_{2}$ production, and inhibiting NF- $\kappa \mathrm{B}$ activation. We suggest that myricetin may be an alternative treatment for gastric injury caused by alcohol intake.
\end{abstract}

\section{Introduction}

Gastric ulcer (also known as peptic ulcer) is a major gastrointestinal disorder whose global incidence and prevalence are on the rise. The prevalence of gastric ulcer is estimated at $0.2-0.5 \%$ in Western countries and $2-3 \%$ in Asian countries, and recurrence rates are as high as $60 \%$ [1]. The lesion has multiple etiologies associated with perturbation of the balance between factors that seek to protect or damage the mucosal epithelium [2]. The damaging factors can include overuse of nonsteroidal anti-inflammatory drugs (NSAIDs), smoking, Helicobacter pylori (H. pylori) infection, alcohol consumption, and psychological and physiological stress [3]. Among them, excessive alcohol drinking is considered to be the leading cause of gastric mucosal damage [4].
The strategies currently used to treat gastric ulcer are based on the cause of the ulcer; they include the use of proton-pump inhibitors, antibiotics aimed at eradicating H. pylori infection (if present), and the application of histamine type- 2 receptor blockers that reduce acid production [5]. Stomach acid-neutralizing antacids and cytoprotective agents, such as sucralfate and misoprostol, may also be employed. However, many of these drugs can have undesirable adverse effects and impose a cost burden on gastric ulcer patients $[6,7]$. Therefore, researchers continue to seek new agents that can be used to treat gastric ulcer with greater safety, fewer side effects, higher efficiency, and lower cost.

Myricetin (3,5,7-trihydroxy-2-(3,4,5-trihydroxyphenyl) chromen-4-one) is a natural flavonoid that belongs to the flavonol subclass and is commonly found in vegetables, fruits, berries, medicinal herbs, and tea [8]. It is particularly 
notable in certain natural products, including fresh broad beans (Phaseolus vulgaris), black tea, broccoli (Brassica oleracea), bell pepper (Capsicum annuum), and garlic (Allium sativum) [9]. Myricetin has multiple biological properties, including antioxidant [10], antimicrobial [11], antiviral [12], anti-inflammatory [13], antitumor [14], analgesic [15], hepatoprotective [16], hypoglycemic [17], hypolipidemic [18], cardioprotective [19], and neurological damage-inhibiting [20] actions. Myricetin suppresses gastric $\mathrm{H}^{+}, \mathrm{K}^{+}$-ATPase in the gastrointestinal tract and thus could be a useful starting compound for developing new agents to alleviate gastric acid secretion [21]. In addition, myricetin has been shown to prevent ulcerative colitis and colorectal tumor in dextran sulfate sodium- (DSS-) induced model mice $[22,23]$. Based on the previous reports, we hypothesized that myricetin may have antiulcerogenic potential in the stomach. Here, we investigated the protective effect of myricetin in a rat model of ethanol-induced gastric injury.

\section{Materials and Methods}

2.1. Chemical. We obtained myricetin (CAS No. 529-44-2; purity, 97.8\%) from Tokyo Chemical Industry Co., Ltd. (Tokyo, Japan).

2.2. Animals. Male 6- to 7-week-old Sprague Dawley (SD) rats weighing 150-250 g were provided by Orient Bio (Republic of Korea). Animals were raised in a constant temperature $\left(23 \pm 2^{\circ} \mathrm{C}\right)$ with a regular $12: 12$-h light: dark cycle and acclimated for 1 week prior to experiments, as previously described [2, 24, 25]. Animal experiments were approved by the Animal Experimental Ethics Committee of Chungnam National University (approval number: CNU00444) and were performed in compliance with the National Institutes of Health Guide for the Care and Use of Laboratory Animals.

2.3. Experimental Design. Gastric lesions were induced by oral administration of absolute ethanol, as previously described $[2,24,25]$. Rats were randomly divided into the following four groups (Table $1 ; n=6-7$ per group): normal control (NC), ethanol (EtOH), omeprazo$\mathrm{le}+\mathrm{EtOH} \quad(\mathrm{Ome}+\mathrm{EtOH})$, and myricetin $+\mathrm{EtOH}$ $(\mathrm{Myr}+\mathrm{EtOH})$. Animals were fasted for $24 \mathrm{~h}$ prior to induction. Rats of the NC group were given $5 \mathrm{~mL} / \mathrm{kg}$ body weight (b.w.) phosphate-buffered saline (PBS; vehicle), while those in the $\mathrm{EtOH}, \mathrm{Ome}+\mathrm{EtOH}$, and $\mathrm{Myr}+\mathrm{EtOH}$ groups were given the same volume $(5 \mathrm{~mL} / \mathrm{kg}$ b.w.) of absolute ethanol by oral gavage. At $1 \mathrm{~h}$ prior to alcohol administration, the Ome + EtOH group was given an oral dose of omeprazole (20 mg/kg b.w.) and the $\mathrm{Myr}+\mathrm{EtOH}$ group was given myricetin $(12 \mathrm{mg} / \mathrm{kg}$ b.w.). The dose of myricetin was determined according to a previous dosing experiment of ethanol-induced ulceration on rats. At $1 \mathrm{~h}$ after ethanol treatment, all animals were sacrificed by overdoses of $50 \mathrm{mg} / \mathrm{kg}$ pentobarbital, and stomachs were collected.
2.4. Macroscopic Determination of Gastric Ulcer Area. Stomachs were collected and dissected along the greater curvature, and the gastric mucosa was rinsed with normal saline. Stomach tissues were flattened and photographed. Image analysis software (ImageJ 46a; NIH, USA) was used to analyze the ulcerated area (UA; $\mathrm{mm}^{2}$ ), and the ratio of UA to gastric area and the inhibition percentage were analyzed. The inhibition percentage was calculated as follows: ((UA control - UA treated)/UA control)) $\times 100 \%$.

2.5. Histopathological Examination. Stomach tissues were fixed in $10 \%$ neutral buffered formalin (NBF), paraffinembedded by routine histopathological methods, sectioned at $4 \mu \mathrm{m}$, and stained with hematoxylin and eosin (H\&E).

2.6. Determination of Malondialdehyde (MDA), Total Glutathione (GSSG/GSH), and Superoxide Dismutase (SOD). To analyze MDA, stomach samples were prepared in PBS with butylated hydroxytoluene (BHT) to prevent further oxidation. The tissue homogenates were centrifuged at $10,000 \mathrm{~g}$ for $5 \mathrm{~min}$, supernatants were collected, and MDA levels were determined using a commercially available kit (Cell Biolabs, USA). The levels were normalized based on the total protein concentration of each lysate. For assessment of total glutathione, tissue lysates were prepared in 5\% metaphosphoric acid (MPA) and centrifuged at 12,000 rpm for $15 \mathrm{~min}$ at $4^{\circ} \mathrm{C}$, and total glutathione was assessed using a commercially available kit (Cell Biolabs) and normalized by the protein concentration. For activity assessment of the antioxidative enzyme, SOD, stomach tissues were homogenized in PBS and centrifuged, and collected supernatants were assessed for SOD activity using a commercially available kit (Cayman, USA). The results were normalized by the protein concentration and are expressed as $\mathrm{U} / \mathrm{mg}$ protein. The protein concentrations used for normalization were determined by the Bradford method.

2.7. Determination of Prostaglandin $E_{2}\left(P G E_{2}\right)$. Stomach tissue samples were homogenized and centrifuged, and supernatants were assessed for $\mathrm{PGE}_{2}$ using a commercially available kit (Cayman, USA). Absorbance was measured at $450 \mathrm{~nm}$ with an ELISA microplate reader (Bio-Rad Laboratories, USA).

2.8. Western Blot Analysis. Stomach tissues were dissected and ground in radioimmunoprecipitation assay (RIPA) buffer (Cell Signaling Technology, USA) containing phosphatase and protease inhibitors (Roche, Germany). The samples were centrifuged, and supernatants were collected. The protein content was analyzed by the Bradford method. Tissue proteins were separated by $8 \%$ sodium dodecyl sulfate-polyacrylamide gel electrophoresis (SDS-PAGE), transferred to a polyvinylidene fluoride (PVDF) membrane (Millipore, USA) at $350 \mathrm{~mA}$ for $2 \mathrm{~h}$, blocked by incubation with $5 \%$ bovine serum albumin (BSA) in PBS containing $0.1 \%$ Tween 20 (PBS-T) for $1 \mathrm{~h}$, and then incubated overnight with primary antibodies at $4^{\circ} \mathrm{C}$. The utilized antibodies 
TABLE 1: Animal study group design.

\begin{tabular}{lc}
\hline Group & Treatment (dose) \\
\hline $\mathrm{NC}$ & PBS $(5 \mathrm{~mL} / \mathrm{kg}$ body weight $)+\mathrm{PBS}(5 \mathrm{~mL} / \mathrm{kg}$ body weight $)$ \\
$\mathrm{EtOH}$ & PBS $(5 \mathrm{~mL} / \mathrm{kg}$ body weight $)+$ ethanol $(5 \mathrm{~mL} / \mathrm{kg}$ body weight $)$ \\
$\mathrm{Ome}+\mathrm{EtOH}$ & Omeprazole $(20 \mathrm{mg} / \mathrm{kg}$ body weight $)+$ ethanol $(5 \mathrm{~mL} / \mathrm{kg}$ body weight $)$ \\
$\mathrm{Myr}+\mathrm{EtOH}$ & Myricetin $(20 \mathrm{mg} / \mathrm{kg}$ body weight $)+$ ethanol $(5 \mathrm{~mL} / \mathrm{kg}$ body weight $)$ \\
\hline
\end{tabular}

recognized phospho-nuclear factor kappa B (p-NF- $\kappa$ B), NF$\kappa \mathrm{B}$ (Cell Signaling Technology), and $\beta$-actin (Sigma-Aldrich, USA). After being washed with PBS-T, membranes were exposed to rabbit or mouse secondary antibodies, as appropriate, for $2 \mathrm{~h}$ and developed using a western blot chemiluminescent substrate (Thermo Scientific, USA).

2.9. RNA Isolation and Quantitative Real-Time PCR Analysis. RNA was isolated with TRIzol reagent (Invitrogen, USA) in accordance with the manufacturer's protocol. The level of RNA was assessed by the intensity at $260 \mathrm{~nm}$, and its purity was determined based on the intensity ratio of $260 \mathrm{~nm}$ to $280 \mathrm{~nm}$. RNA was reverse transcribed (ReverTra Ace kit, Toyobo, Japan), and the expression levels of the mRNAs of interest were measured using a StepOnePlus Real-Time PCR System (Applied Biosystems, USA). The relative transcript level was calculated from duplicate samples after the results were normalized by the mRNA expression of glyceraldehyde-3phosphate dehydrogenase (GAPDH). The utilized primer pairs and PCR condition are listed in Table 2. PCR data ware expressed as the fold change of the target gene relative to the level of GAPDH, as calculated using the $2^{-\Delta \Delta C t}$ method.

2.10. Statistical Analysis. Data are expressed as mean$\mathrm{s} \pm$ standard deviation (SD). Statistical significance was determined using one-way analysis of variance (ANOVA), and the significance between the groups was analyzed by Tukey's multiple comparison test. $P$ values $<0.05$ were considered significant.

\section{Results}

3.1. Effect of Myricetin on Ethanol-Induced Acute Gastric Injury. No injury was found in the normal control group (Figure 1(a)). Severe ulcers accompanied by elongated-band hemorrhage were detected in the glandular layer of the stomach in rats from the ethanol-administered group (Figure 1(b)), but this ethanol-induced gastric injury was attenuated in the omeprazole- and myricetin-pretreated groups (Figures 1(c) and 1(d)). The UA in the alcoholtreated group was $174.3 \pm 41.7 \mathrm{~mm}^{2}$, whereas that in rats pretreated with omeprazole or myricetin was $54.0 \pm 42.8 \mathrm{~mm}^{2}$ (69\% inhibition) and $74.2 \pm 19.1 \mathrm{~mm}^{2}$ (57\% inhibition), respectively (Table 3). Histopathological analyses showed severe hemorrhage, extensive submucosal edema, and the loss of gastric mucosa from stomach tissues of ethanol-treated rats (Figure 2(b)), but this ethanol-induced damage was notably reduced by omeprazole or myricetin pretreatment (Figures 2(c) and 2(d)). These results indicate that myricetin significantly decreased alcohol-induced gastric ulcer and injury in the rat model.
3.2. Effect of Myricetin on Lipid Peroxidation and Antioxidant Enzymes. The MDA level in stomach tissue was markedly enhanced in ethanol-treated rats compared to the normal control group. Omeprazole or myricetin pretreatment significantly decreased this ethanol-induced increase in MDA (Figure 3(a)). The gastric total glutathione concentration and SOD activity were lower in the ethanol-treated group than in the normal control group, but omeprazole or myricetin pretreatment significantly attenuated these decreases, such that the total glutathione concentration and SOD activity were higher in the omeprazole- and myricetin-pretreated groups than the ethanol-treated group (Figures 3(b) and 3(c)). These findings suggest that myricetin significantly alleviated the oxidative stress induced by ethanol administration.

3.3. Effect of Myricetin on Activation of NF- $\kappa B$. Western blot analyses showed that phosphorylation of NF- $\kappa$ B in stomach tissues was increased in the ethanol-treated group compared with the normal control group, but omeprazole or myricetin pretreatment significantly attenuated NF- $\kappa \mathrm{B}$ activity compared with the ethanol-treated group (Figure 4). This indicates that myricetin exerts protective activity through suppression of the NF- $\kappa \mathrm{B}$-dependent pathway.

3.4. Effect of Myricetin on the Expression of Cyclooxygenase-1 $(C O X-1)$ and $P G E_{2}$. The mRNA expression level of COX-1 was elevated after ethanol administration, and this increase was significantly enhanced by pretreatment with omeprazole or myricetin (Figure 5(a)). The level of $\mathrm{PGE}_{2}$ was slightly lower in rats subjected to intragastric administration of ethanol compared with the normal control group, but this level was significantly increased by pretreatment with omeprazole or myricetin (Figure 5(b)). These results suggest that myricetin might show protective effects on ethanolinduced gastric injury at least partially via COX-1 and $\mathrm{PGE}_{2}$.

\section{Discussion}

Myricetin is a flavonoid that offers numerous pharmacological benefits, such as antioxidant, anti-inflammatory, and anticancer activities $[10,13]$. Here, we assessed the potential pharmacologic effects of myricetin against ethanol-induced gastric mucosal injury in SD rats. We found that myricetin pretreatment protected gastric tissue against acute injury induced by absolute alcohol, suppressing gastric injury, lipid peroxidation, and NF- $\kappa \mathrm{B}$ activation while increasing antioxidant activity and $\mathrm{PGE}_{2}$ expression.

Ethanol-induced stomach ulceration is a classic model that is often used in studies assessing the protective effects of drugs in the stomach, particularly cytoprotective and/or 
TABLe 2: Primer pairs used for qRT-PCR.

\section{Primer sequence}

Cox-1

Gapdh
F: 5' ${ }^{\prime}$-TGACTATCTGGCGGGTGACT-3'

R: $5^{\prime}$-CTTGCTGGACATTGGGTTCT-3'

F: $5^{\prime}$-ACAGCAACAGGGTGGTGGAC- $3^{\prime}$ R: 5'-TTTGAGGGTGCAGCGAACTT-3'

Real-time PCR condition

Step $\quad$ Temperature $\left({ }^{\circ} \mathrm{C}\right)$

Initial denaturation

Denaturation

Annealing and Extension

Melt curve stage

95

95

60

95

60
Time

$10 \mathrm{~min}$

$15 \mathrm{sec}$

$1 \mathrm{~min}$

$15 \mathrm{sec}$

$1 \mathrm{~min}$
Cycle

1

40

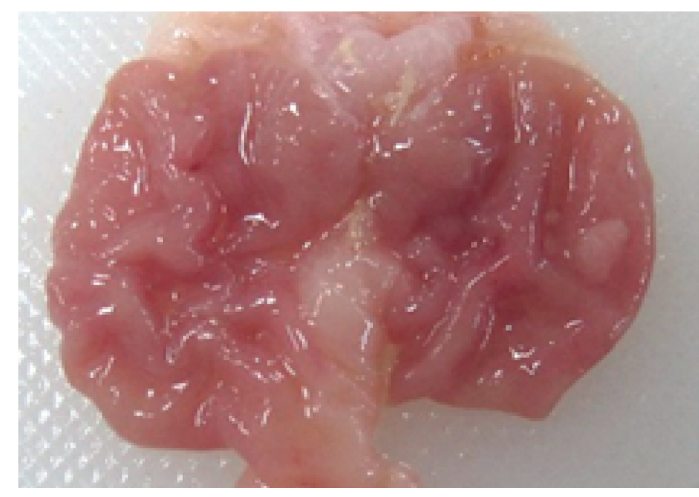

(a)

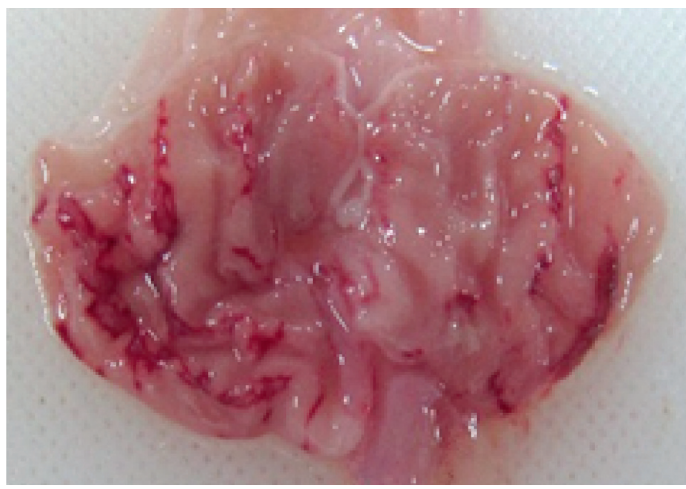

(c)

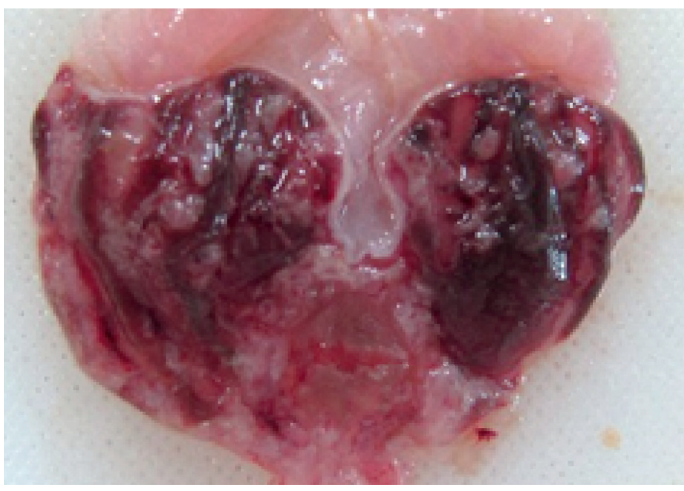

(b)

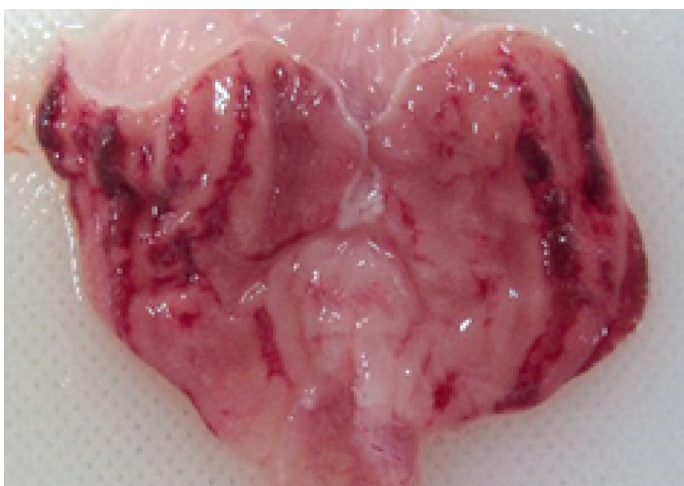

(d)

FIgURE 1: Gross findings of the gastric mucosa. (a) Normal control group. (b) Ethanol-treated group. (c) Omeprazole plus ethanol-treated group. (d) Myricetin plus ethanol-treated group.

TABLE 3: Effects of myricetin on gastric ulcer area $\left(\mathrm{UA}, \mathrm{mm}^{2}\right)$ and percentage inhibition.

\begin{tabular}{lccc}
\hline Group & Treatment & UA $\left(\mathrm{mm}^{2}\right)$ & Inhibition $(\%)$ \\
\hline $\mathrm{NC}$ & PBS + PBS & $0.0 \pm 0.0$ & - \\
$\mathrm{EtOH}$ & PBS + ethanol & $174.3 \pm 41.7^{\# \#}$ & - \\
$\mathrm{Ome}+\mathrm{EtOH}$ & Omeprazole + ethanol & $54.0 \pm 42.8^{* *}$ & 69 \\
$\mathrm{Myr}+\mathrm{EtOH}$ & Myricetin + ethanol & $74.2 \pm 19.1^{* *}$ & 57 \\
\hline
\end{tabular}

The results are expressed as mean $\pm \mathrm{SD}$. ${ }^{\# \#} P<0.01$ compared to the NC group. ${ }^{* *} P<0.01$ compared to the EtOH group. 

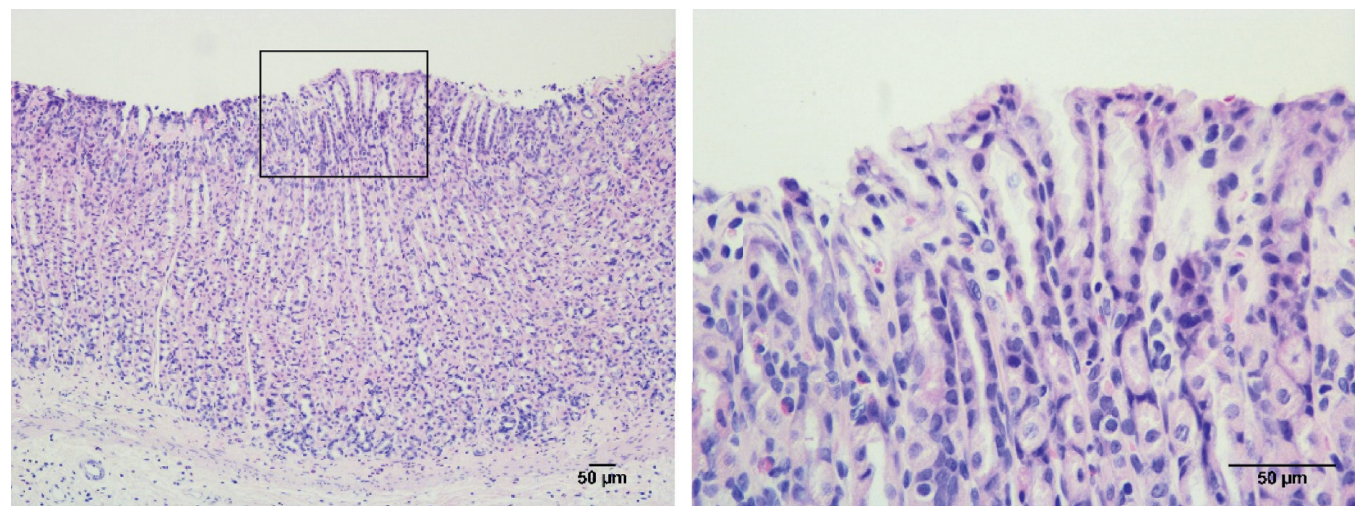

(a)
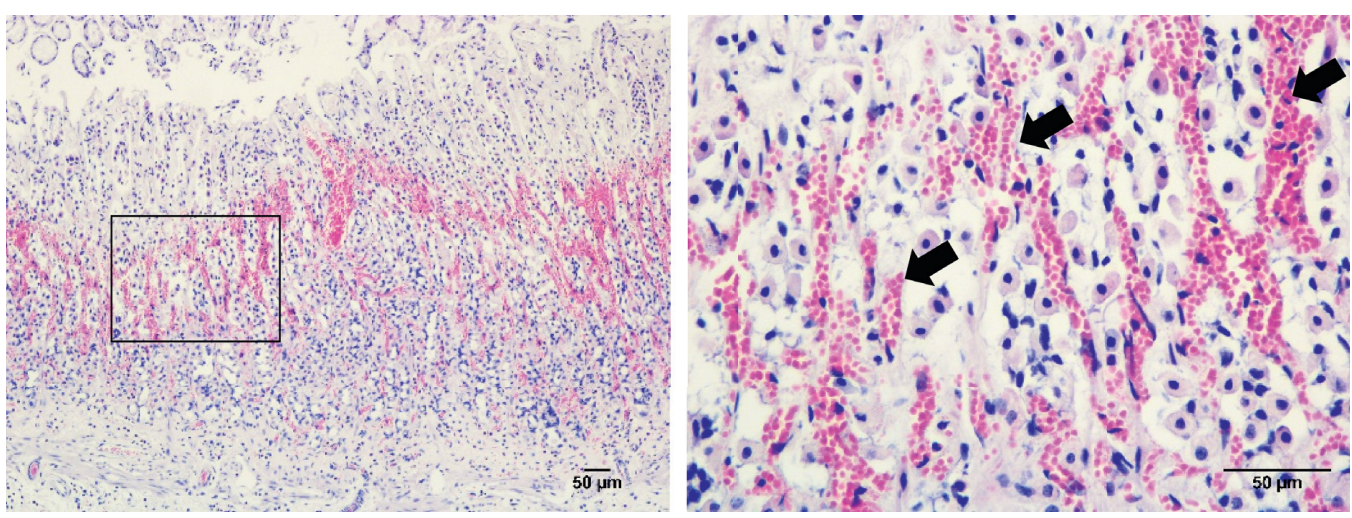

(b)

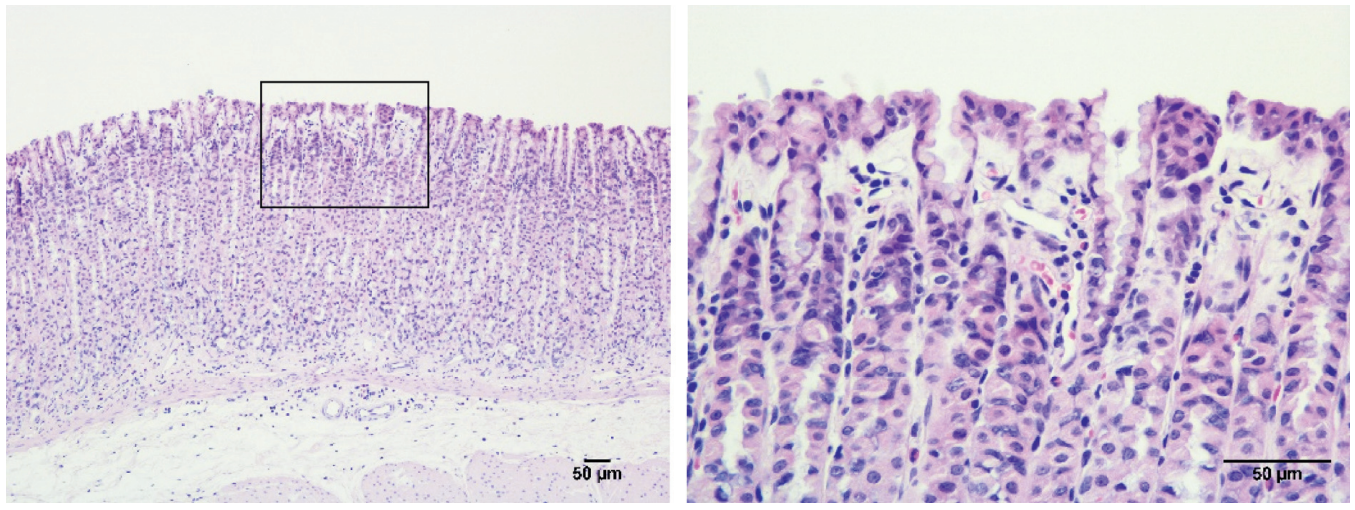

(c)

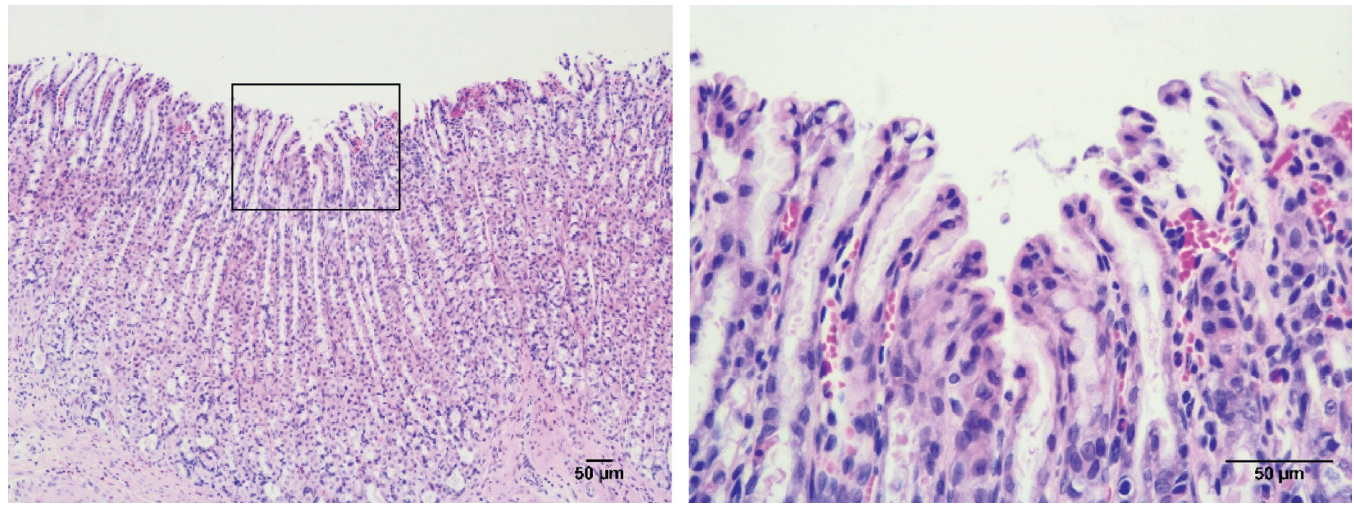

(d)

FIGURE 2: Histopathological examination of gastric tissue. (a) Normal control group. (b) Ethanol-treated group. (c) Omeprazole plus ethanoltreated group. (d) Myricetin plus ethanol-treated group. No disturbance in the gastric mucosa was detected in the normal control group, whereas the ethanol-treated group showed severe destruction of the surface epithelium and necrotic lesions. The black arrow indicates severe hemorrhage of the mucosal layer. Magnification, $\times 100$ and $\times 400$. 


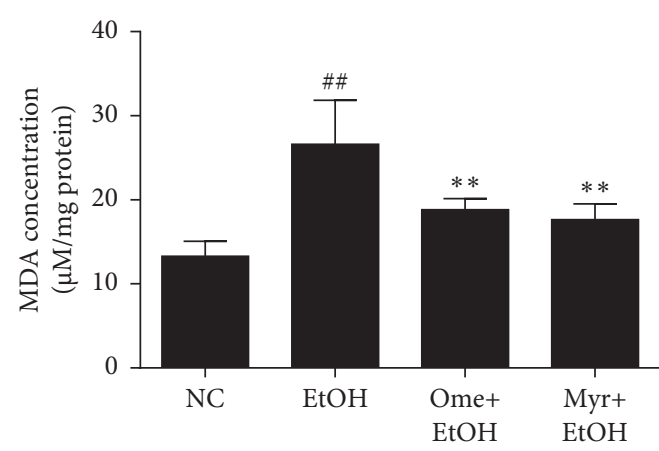

(a)

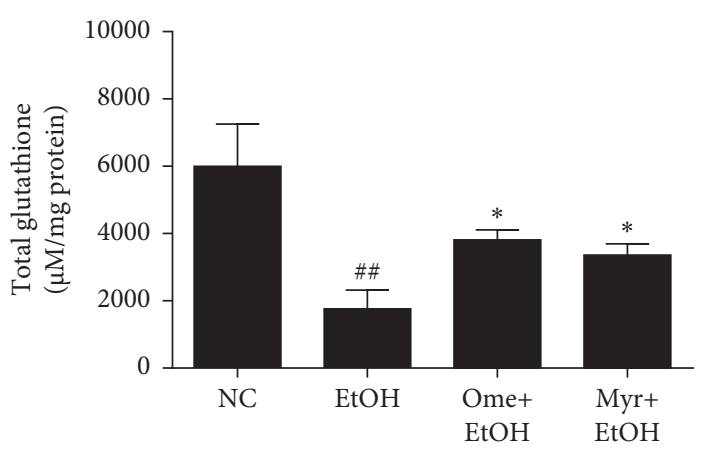

(b)

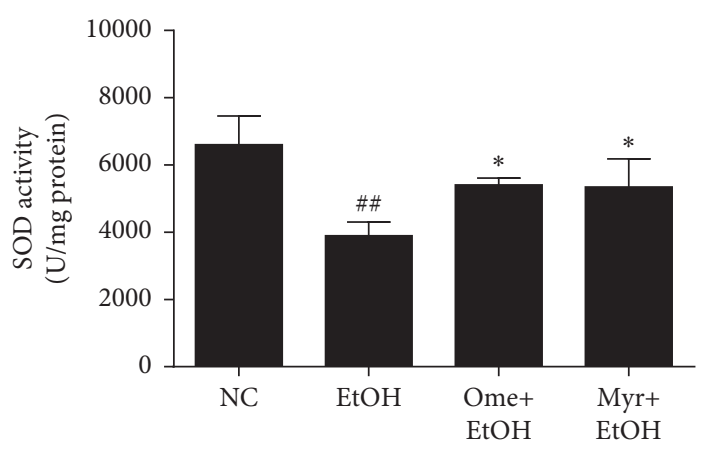

(c)

Figure 3: Levels of MDA, total glutathione (GSSG/GSH), and SOD in stomach tissue. (a) MDA concentration. (b) Total glutathione concentration. (c) SOD activity. Values represent means \pm SD $\left({ }^{\#} P<0.05\right.$ and ${ }^{\# \#} P<0.01$ compared to the normal control group; ${ }^{*} P<0.05$ and ${ }^{* *} P<0.01$ compared to the ethanol-treated group).
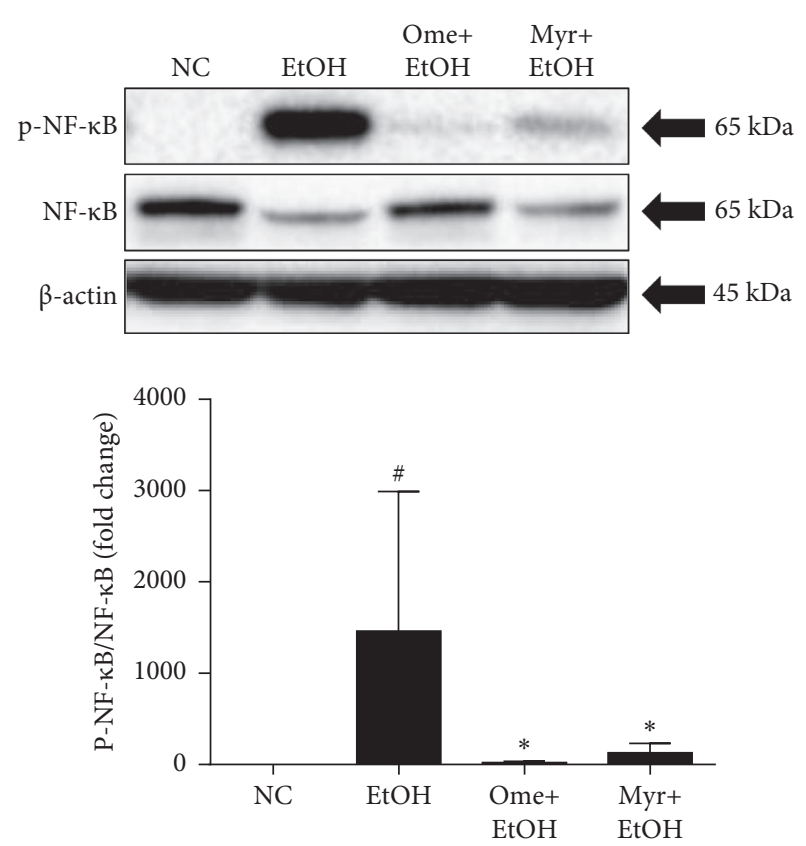

Figure 4: NF- $\kappa$ B activity. Values represent means \pm SD $\left({ }^{\#} P<0.05\right.$ and ${ }^{\# \#} P<0.01$ compared to the normal control group; ${ }^{*} P<0.05$ and ${ }^{* *} P<0.01$ compared to the ethanol-treated group).

antioxidant activities [26, 27]. Upon administration, ethanol rapidly penetrates the gastric mucosa; this exposes the mucosal layer to hydrochloric acid and pepsin [28], which have proteolytic and hydrolytic effects that damage cell membranes to cause cell exfoliation, erosion, and ulcer [29]. Here, we show that myricetin pretreatment inhibited ulceration by $\sim 57 \%$ in this model and thus showed an efficacy similar to that of omeprazole. The antiulcer effect of myricetin was supported by our histopathological examination, which revealed that myricetin-administered rats had intact mucosal structures and glandular elements, along with decreases in submucosal edema and hemorrhage. These results indicate that myricetin protects the stomach against ethanol-induced injury to the gastric mucosa.

Gastric lesions induced by alcohol are closely related to the generation of reactive oxygen species (ROS), reflecting that lesions are associated with an imbalance between oxidative and antioxidative cellular processes [30]. This shift to an oxidative state arises from the release of hydroperoxy free radicals and superoxide anions, whose increases cause an oxidative stress that can be visualized as an increased level of MDA [31,32]. MDA is generated by the peroxidation of polyunsaturated fatty acids or related esters within cell membranes and is used as a marker of ROS-induced cellular damage [33]. GSH and SOD are among the defense mechanisms that protect cells against ROS-induced lipid peroxidation [34]. GSH inhibits lipid peroxidation and scavenges radicals; it is among the most abundant cellular antioxidants and acts to detoxify hydrogen peroxide by various glutathione peroxidases [35]. SOD, meanwhile, acts on superoxide produced during oxidative stress, converting it to hydrogen peroxide [36]. In the present study, oral 


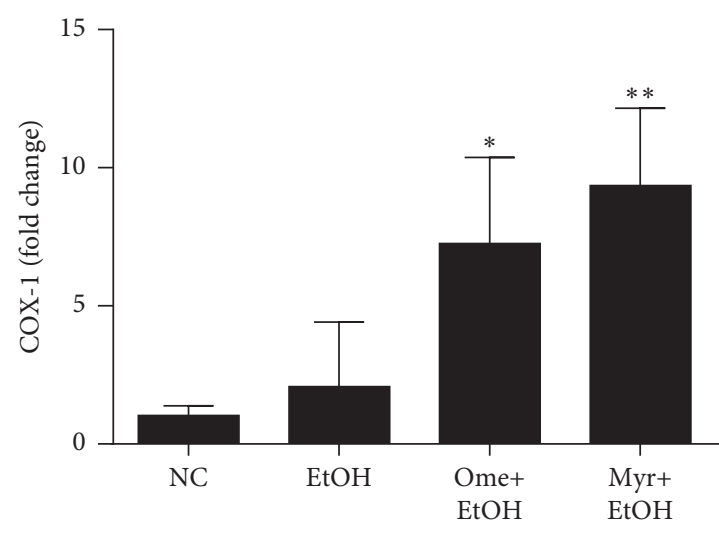

(a)

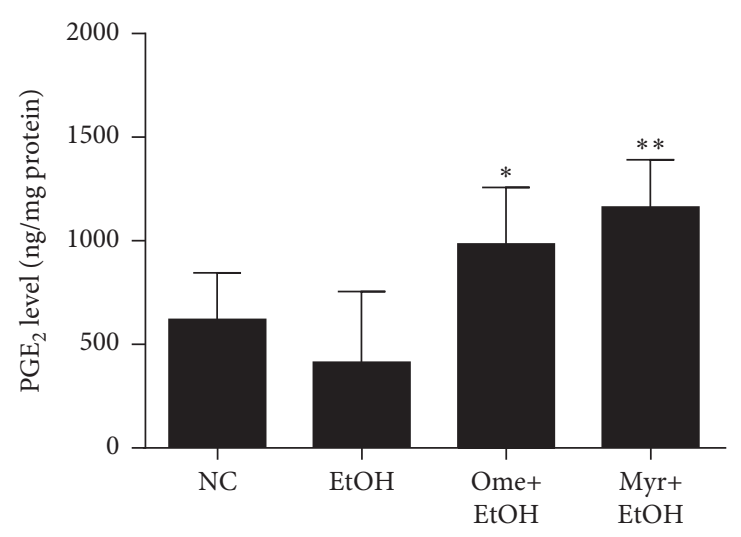

(b)

FIgURE 5: Expression of COX-1 and production of $\mathrm{PGE}_{2}$. (a) COX-1 mRNA levels. (b) $\mathrm{PGE}_{2}$ levels. Values represent means $\pm \mathrm{SD}\left({ }^{\#} P<0.05\right.$ and ${ }^{\# \#} P<0.01$ compared to the normal control group; ${ }^{*} P<0.05$ and ${ }^{* *} P<0.01$ compared to the ethanol-treated group).

administration of alcohol markedly increased the levels of MDA and decreased those of total glutathione and SOD activity, supporting the notion that oxidative stress plays an important role in the pathogenesis of ethanol-induced gastric injury. Pretreatment with myricetin markedly increased the level of SOD and total glutathione and decreased that of MDA. These findings are consistent with previous reports that myricetin exerts a strong antioxidant activity by scavenging hydroxyl free radicals [37] and can restore cellular antioxidant defense enzymes, including SOD, catalase (CAT), and glutathione peroxidase (GPx) [38]. Taken together, our results indicate that myricetin appears to exert a gastroprotective effect via an antioxidant mechanism.

ROS activates NF- $\kappa \mathrm{B}$, which is a critical regulator that promotes the transcription of genes related to inflammation, cell survival, and immune responses. During ethanol-induced gastric ulcer formation, NF- $\kappa$ B governs the increased transcription of genes encoding inducible nitric oxide synthase (iNOS), COX-2, and inflammatory cytokines including tumor necrosis factor- $\alpha$ (TNF- $\alpha$ ), interleukin (IL)-6, and IL- $1 \beta$ [2]. NF- $\kappa$ B expression is therefore a crucial nexus for gastric ulcer formation, making it a logical target for the treatment of inflammatory conditions [39]. Here, we found that alcohol administration induced a remarkable increase of NF- $\kappa$ B that was significantly suppressed by pretreatment with myricetin. These results are consistent with previous reports that myricetin exerts anti-inflammatory activity by suppressing $\mathrm{I} \kappa \mathrm{B}$ kinase/NF- $\kappa \mathrm{B}$-dependent signaling $[40,41]$.

Gastric mucosal cells produce prostaglandins (PGs), which function as important endogenous mediators during acute gastric mucosal injury $[42,43]$ and contribute to mucosal defense by regulating the secretion of acid and stimulating those of mucus, bicarbonate, and phospholipids. PGs also accelerate epithelial restitution and mucosal healing [44] and mediate inflammatory reactions by increasing vascular permeability, leading to vasodilation and increased blood flow [45]. PGs are generated by COX enzymes; among them, COX-1 produces PGs that play essential roles in homeostatic functions, including maintenance of mucosal integrity and mucosal blood flow
[46]. Here, we found that the levels of COX-1 and $\mathrm{PGE}_{2}$ (the most abundant prostaglandin [47]) were significantly higher in the myricetin-pretreated group than in the alcoholtreated group. This suggests that myricetin acts via COX-1 and $\mathrm{PGE}_{2}$ to exert protective effects against ethanol-induced gastric mucosal injury.

We herein used omeprazole, a proton-pump inhibitor that suppresses gastric acid secretion [5], as a positive control. As omeprazole can produce undesirable adverse effects and impose a cost burden on gastric ulcer patient $[6,7]$, there is increasing demand for alternative strategies that can treat gastrointestinal diseases with fewer adverse effects. Myricetin was previously shown to inhibit $\mathrm{H}^{+}, \mathrm{K}^{+}$-ATPase and attenuate gastric acid secretion, suggesting its potential to alleviate gastric acid-related diseases when used alone or in a combination treatment [21]. The present study demonstrates the first evidence that myricetin may have gastroprotective effects against alcohol-induced gastric ulcer. The gastroprotective effects of myricetin appear to be at least partially mediated by its ability to reduce oxidative stress, inhibit NF- $\kappa \mathrm{B}$, and stimulate $\mathrm{PGE}_{2}$ secretion. Further studies are needed to confirm that myricetin is a viable alternative for the clinical management of gastric ulcer diseases.

\section{Conclusion}

Our study results indicate that myricetin effectively inhibits ethanol-induced acute gastric injury by preventing oxidative damage, stimulating $\mathrm{PGE}_{2}$ production, and inhibiting NF$\kappa \mathrm{B}$ activation. Myricetin may be an alternative treatment for gastric injury caused by alcohol intake.

\section{Data Availability}

The data generated from the findings of this study are included within the article.

\section{Conflicts of Interest}

The authors declare that they have no conflicts of interest. 


\section{Acknowledgments}

This work was supported by a research scholarship of Chungnam National University.

\section{References}

[1] A. Wang, J. Yerxa, S. Agarwal et al., "Surgical management of peptic ulcer disease," Current Problems in Surgery, vol. 57, no. 2, Article ID 100728, 2020.

[2] J.-W. Song, C.-S. Seo, T.-I. Kim et al., "Protective effects of Manassantin A against ethanol-induced gastric injury in rats," Biological \& Pharmaceutical Bulletin, vol. 39, no. 2, pp. 221229, 2016.

[3] K. Toljamo, S. Niemelä, A.-L. Karvonen, R. Karttunen, and T. J. Karttunen, "Histopathology of gastric erosions association with etiological factors and chronicity," Helicobacter, vol. 16, no. 6, pp. 444-451, 2011.

[4] A. Franke, S. Teyssen, and M. V. Singer, "Alcohol-related diseases of the esophagus and stomach," Digestive Diseases (Basel, Switzerland), vol. 23, no. 3-4, pp. 204-213, 2005.

[5] A. Lanas and F. K. L. Chan, "Peptic ulcer disease," The Lancet, vol. 390, no. 10094, pp. 613-624, 2017.

[6] F. K. Chan and W. Leung, "Peptic-ulcer disease," The Lancet, vol. 360, no. 9337, pp. 933-941, 2002.

[7] K. S. Jain, A. K. Shah, J. Bariwal et al., "Recent advances in proton pump inhibitors and management of acid-peptic disorders," Bioorganic \& Medicinal Chemistry, vol. 15, no. 3, pp. 1181-1205, 2007.

[8] D. Semwal, R. Semwal, S. Combrinck, and A. Viljoen, "Myricetin: a dietary molecule with diverse biological activities," Nutrients, vol. 8, no. 2, p. 90, 2016.

[9] J. A. Ross and C. M. Kasum, "Dietaryflavonoids: bioavailability, metabolic effects and safety," Annual Review of $\mathrm{Nu}$ trition, vol. 22, no. 1, pp. 19-34, 2002.

[10] S. M. Hassan, M. M. Khalaf, S. A. Sadek, and A. M. AboYoussef, "Protective effects of apigenin and myricetin against cisplatin-induced nephrotoxicity in mice," Pharmaceutical Biology, vol. 55, no. 1, pp. 766-774, 2017.

[11] R. Ren, S. Yin, B. Lai et al., "Myricetin antagonizes semenderived enhancer of viral infection (SEVI) formation and influences its infection-enhancing activity," Retrovirology, vol. 15 , no. 1 , p. $49,2018$.

[12] J. T. Ortega, A. I. Suárez, M. L. Serrano, J. Baptista, F. H. Pujol, and H. R. Rangel, "The role of the glycosyl moiety of myricetin derivatives in anti-HIV-1 activity in vitro," AIDS Research and Therapy, vol. 14, no. 1, p. 57, 2017.

[13] W. Hou, S. Hu, Z. Su et al., "Myricetin attenuates LPS-induced inflammation in RAW 264.7 macrophages and mouse models," Future Medicinal Chemistry, vol. 10, no. 19, pp. 2253-2264, 2018.

[14] S. C. Iyer, A. Gopal, and D. Halagowder, "Myricetin induces apoptosis by inhibiting P21 activated kinase 1 (PAK1) signaling cascade in hepatocellular carcinoma," Molecular and Cellular Biochemistry, vol. 407, no. 1-2, pp. 223-237, 2015.

[15] Y. Tong, X.-M. Zhou, S.-J. Wang, Y. Yang, and Y.-L. Cao, "Analgesic activity of myricetin isolated from Myrica rubra Sieb. et Zucc. leaves," Archives of Pharmacal Research, vol. 32, no. 4, pp. 527-533, 2009.

[16] J. Guo, Y. Meng, Y. Zhao, Y. Hu, D. Ren, and X. Yang, "Myricetin derived from Hovenia dulcis thunb. ameliorates vascular endothelial dysfunction and liver injury in high choline-fed mice," Food \& Function, vol. 6, no. 5, pp. 1620-1634, 2015.
[17] N. Kandasamy and N. Ashokkumar, "Protective effect of bioflavonoid myricetin enhances carbohydrate metabolic enzymes and insulin signaling molecules in streptozotocincadmium induced diabetic nephrotoxic rats," Toxicology and Applied Pharmacology, vol. 279, no. 2, pp. 173-185, 2014.

[18] H.-M. Su, L.-N. Feng, X.-D. Zheng, and W. Chen, "Myricetin protects against diet-induced obesity and ameliorates oxidative stress in C57BL/6 mice," Journal of Zhejiang UniversityScience B, vol. 17, no. 6, pp. 437-446, 2016.

[19] R. Tiwari, M. Mohan, S. Kasture, A. Maxia, and M. Ballero, "Cardioprotective potential of myricetin in isoproterenolinduced myocardial infarction in Wistar rats," Phytotherapy Research, vol. 23, no. 10, pp. 1361-1366, 2009.

[20] Y. Chang, C.-Y. Chang, S.-J. Wang, and S.-K. Huang, "Myricetin inhibits the release of glutamate in rat cerebrocortical nerve terminals," Journal of Medicinal Food, vol. 18, no. 5, pp. 516-523, 2015.

[21] Y. Miyazaki, A. Ichimura, S. Sato et al., "The natural flavonoid myricetin inhibits gastric $\mathrm{H}^{+}, \mathrm{K}^{+}$-ATPase," European Journal of Pharmacology, vol. 820, pp. 217-221, 2018.

[22] X. Qu, Q. Li, Y. Song et al., "Potential of myricetin to restore the immune balance in dextran sulfate sodium-induced acute murine ulcerative colitis," Journal of Pharmacy and Pharmacology, vol. 72, no. 1, pp. 92-100, 2020.

[23] F. Wang, Z.-Y. Song, X.-J. Qu et al., "M10, a novel derivative of Myricetin, prevents ulcerative colitis and colorectal tumor through attenuating robust endoplasmic reticulum stress," Carcinogenesis, vol. 39, no. 7, pp. 889-899, 2018.

[24] I.-S. Shin, W.-Y. Jeon, H.-K. Shin, S.-W. Cha, and M.-Y. Lee, "Banhabaekchulchunma-tang, a traditional herbal formula attenuates absolute ethanol-induced gastric injury by enhancing the antioxidant status," BMC Complementary and Alternative Medicine, vol. 13, no. 1, p. 170, 2013.

[25] W.-Y. Jeon, I.-S. Shin, H.-K. Shin, and M.-Y. Lee, "Gastroprotective effect of the traditional herbal medicine, Sipjeondaebo-tang water extract, against ethanol-induced gastric mucosal injury," BMC Complementary and Alternative Medicine, vol. 14, no. 1, p. 373, 2014.

[26] I.-M. Boutemine, M. Amri, Z.-C. Amir et al., "Gastro-protective, therapeutic and anti-inflammatory activities of Pistacia lentiscus $L$. fatty oil against ethanol-induced gastric ulcers in rats," Journal of Ethnopharmacology, vol. 224, pp. 273-282, 2018.

[27] T. Balan, M. H. M. Sani, S. H. Mumtaz Ahmad, V. Suppaiah, N. Mohtarrudin, and Z. A. Zakaria, "Antioxidant and anti-inflammatory activities contribute to the prophylactic effect of semi-purified fractions obtained from the crude methanol extract of Muntingia calabura leaves against gastric ulceration in rats," Journal of Ethnopharmacology, vol. 164, pp. 1-15, 2015.

[28] P. J. Oates and J. P. Hakkinen, "Studies on the mechanism of ethanol-induced gastric damage in rats," Gastroenterology, vol. 94, no. 1, pp. 10-21, 1988.

[29] S. Shaw, V. Herbert, N. Colman, and E. Jayatilleke, "Effect of ethanol-generated free radicals on gastric intrinsic factor and glutathione," Alcohol, vol. 7, no. 2, pp. 153-157, 1990.

[30] F. M. de-Faria, A. C. Almeida, A. Luiz-Ferreira et al., "Antioxidant action of mangrove polyphenols against gastric damage induced by absolute ethanol and ischemia-reperfusion in the rat," Scientific World Journal, vol. 2012, Article ID 327071, 9 pages, 2012.

[31] G. B. Glavin and S. Szabo, "Experimental gastric mucosal injury: laboratory models reveal mechanisms of pathogenesis and new therapeutic strategies," The FASEB Journal, vol. 6, no. 3, pp. 825-831, 1992. 
[32] M. G. Repetto and S. F. Llesuy, "Antioxidant properties of natural compounds used in popular medicine for gastric ulcers," Brazilian Journal of Medical and Biological Research, vol. 35, no. 5, pp. 523-534, 2002.

[33] H. M. A. Sidahmed, A. H. S. Azizan, S. Mohan et al., "Gastroprotective effect of desmosdumotin C isolated from Mitrella kentii against ethanol-induced gastric mucosal hemorrhage in rats: possible involvement of glutathione, heatshock protein-70, sulfhydryl compounds, nitric oxide, and anti-helicobacter pylori activity," BMC Complementary and Alternative Medicine, vol. 13, no. 1, p. 183, 2013.

[34] J. M. MatÉs, C. Pérez-Gómez, and I. N. De Castro, "Antioxidant enzymes and human diseases," Clinical Biochemistry, vol. 32, no. 8, pp. 595-603, 1999.

[35] J. B. Owen and D. A. Butterfield, "Measurement of oxidized/ reduced glutathione ratio," Methods in Molecular Biology, vol. 648, pp. 269-277, 2010.

[36] S. Marklund and G. Marklund, "Involvement of the superoxide anion radical in the autoxidation of pyrogallol and a convenient assay for superoxide dismutase," European Journal of Biochemistry, vol. 47, no. 3, pp. 469-474, 1974.

[37] A. Barzegar, "Antioxidant activity of polyphenolic myricetin in vitro cell- free and cell-based systems," Molecular Biology Research Communications, vol. 5, no. 2, pp. 87-95, 2016.

[38] Z. H. Wang, K. Ah Kang, R. Zhang et al., "Myricetin suppresses oxidative stress-induced cell damage via both direct and indirect antioxidant action," Environmental Toxicology and Pharmacology, vol. 29, no. 1, pp. 12-18, 2010.

[39] J.-H. Yoo, J.-S. Lee, Y.-S. Lee, S. Ku, and H.-J. Lee, "Protective effect of bovine milk against $\mathrm{HCl}$ and ethanol-induced gastric ulcer in mice," Journal of Dairy Science, vol. 101, no. 5, pp. 3758-3770, 2018.

[40] R.-H. Fu, S.-P. Liu, C.-L. Chu et al., "Myricetin attenuates lipopolysaccharide-stimulated activation of mouse bone marrow-derived dendritic cells through suppression of IKK/ NF- $\kappa \mathrm{B}$ and MAPK signalling pathways," Journal of the Science of Food and Agriculture, vol. 93, no. 1, pp. 76-84, 2013.

[41] B. O. Cho, H. H. Yin, S. H. Park, E. B. Byun, H. Y. Ha, and S. I. Jang, "Anti-inflammatory activity of myricetin from Diospyros lotus through suppression of NF- $\kappa$ B and STAT1 activation and Nrf2-mediated HO-1 induction in lipopolysaccharide-stimulated RAW264.7 macrophages," Bioscience Biotechnology \& Biochemistry, vol. 80, no. 8, pp. 1520-1530, 2016.

[42] S. Szabo, "Mechanisms of gastric mucosal injury and protection," Journal of Clinical Gastroenterology, vol. 13, no. Supplement 2, p. S35, 1991.

[43] K. Kobayashi and T. Arakawa, "Arachidonic acid cascade and gastric mucosal injury, protection and healing: topics of this decade," Journal of Clinical Gastroenterology, vol. 21, pp. S12-S17, 1994.

[44] L. Laine, K. Takeuchi, and A. Tarnawski, "Gastric mucosal defense and cytoprotection: bench to bedside," Gastroenterology, vol. 135, no. 1, pp. 41-60, 2008.

[45] T. J. Williams and M. J. Peck, "Role of prostaglandin-mediated vasodilatation in inflammation," Nature, vol. 270, no. 5637, pp. 530-532, 1977.

[46] J. L. Wallace, W. McKnight, B. K. Reuter, and N. Vergnolle, "NSAID-induced gastric damage in rats: requirement for inhibition of both cyclooxygenase 1 and 2," Gastroenterology, vol. 119, no. 3, pp. 706-714, 2000.

[47] J. Ke, Y. Yang, Q. Che et al., "Prostaglandin E2 (PGE2) promotes proliferation and invasion by enhancing SUMO-1 activity via EP4 receptor in endometrial cancer," Tumor Biology, vol. 37, no. 9, pp. 12203-12211, 2016. 